PREPARED FOR THE U.S. DEPARTMENT OF ENERGY, UNDER CONTRACT DE-AC02-76CH03073

PPPL-3592

PPPL-3592

UC-70

Toroidal Plasma Thruster for Interplanetary and Interstellar Space Flights

by

N.N. Gorelenkov, L.E. Zakharov, and M.V. Gorelenkova

July 2001

NM|

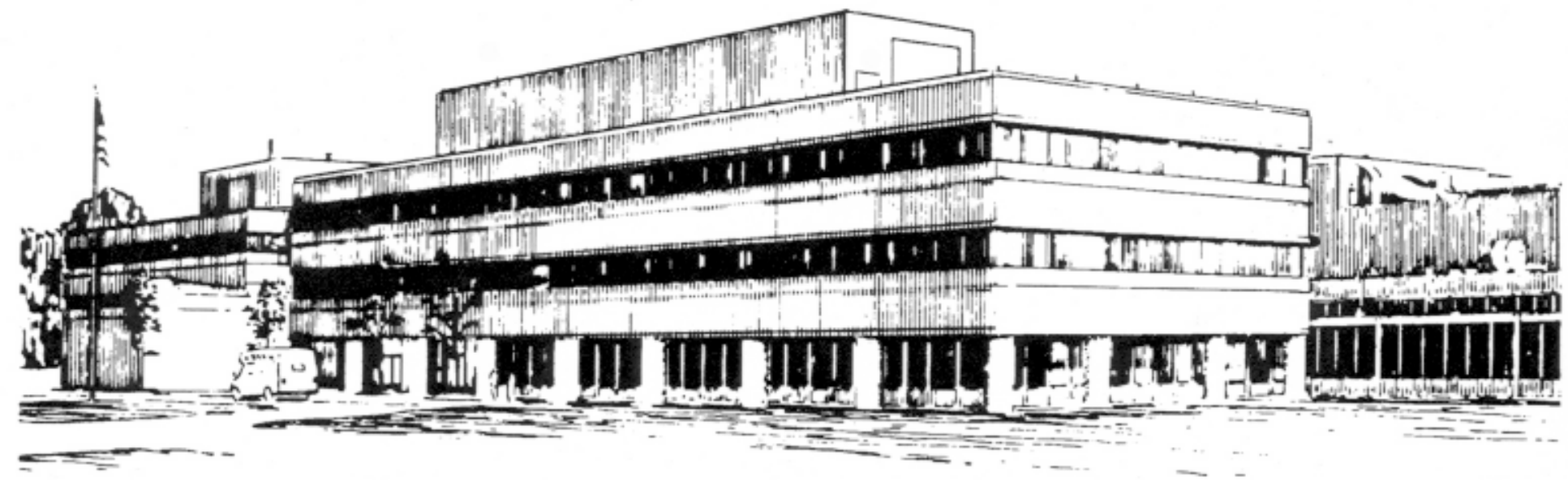

PRINCETON PLASMA PHYSICS LABORATORY PRINCETON UNIVERSITY, PRINCETON, NEW JERSEY 


\section{PPPL Reports Disclaimer}

This report was prepared as an account of work sponsored by an agency of the United States Government. Neither the United States Government nor any agency thereof, nor any of their employees, makes any warranty, express or implied, or assumes any legal liability or responsibility for the accuracy, completeness, or usefulness of any information, apparatus, product, or process disclosed, or represents that its use would not infringe privately owned rights. Reference herein to any specific commercial product, process, or service by trade name, trademark, manufacturer, or otherwise, does not necessarily constitute or imply its endorsement, recommendation, or favoring by the United States Government or any agency thereof. The views and opinions of authors expressed herein do not necessarily state or reflect those of the United States Government or any agency thereof.

\section{Availability}

This report is posted on the U.S. Department of Energy's Princeton Plasma Physics Laboratory Publications and Reports web site in Calendar Year 2001. The home page for PPPL Reports and Publications is: http://www.pppl.gov/pub_report/

DOE and DOE Contractors can obtain copies of this report from:

U.S. Department of Energy

Office of Scientific and Technical Information

DOE Technical Information Services (DTIS)

P.O. Box 62

Oak Ridge, TN 37831

Telephone: (865) 576-8401

Fax: (865) 576-5728

Email: reports@adonis.osti.gov

This report is available to the general public from:

National Technical Information Service

U.S. Department of Commerce

5285 Port Royal Road

Springfield, VA 22161

Telephone: 1-800-553-6847 or

(703) 605-6000

Fax: (703) 321-8547

Internet: http://www.ntis.gov/ordering.htm 


\title{
TOROIDAL PLASMA THRUSTER FOR INTERPLANETARY AND INTERSTELLAR SPACE FLIGHTS
}

\author{
N. N. Gorelenkov ${ }^{\dagger}$, L. E. Zakharov ${ }^{\dagger}$, and M. V. Gorelenkova \\ 9th July 2001 \\ ${ }^{\dagger}$ Princeton Plasma Physics Laboratory, Princeton University, Princeton, NJ, 08543, \\ USA. \\ ${ }^{\ddagger}$ TRINITI, Troitsk, Moscow Region, Russia, 142092.
}

\begin{abstract}
This work represents a conceptual assessment of using the toroidal fusion reactor for the deep space interplanetary and interstellar missions. Toroidal thermonuclear fusion reactors, such as tokamaks and stellarators, are unique for the space propulsion allowing a design with the magnetic configuration localized inside the toroidal magnetic field coils. Plasma energetic ions including charged fusion products can escape such closed configuration at certain condition as a result of the vertical drift in toroidal rippled magnetic field. Escaping particles can be used for the direct propulsion since toroidal drift is directed one way vertically, or to create and heat external confined plasma, so that the later can be used for the propulsion.

D-T fusion neutrons with the energy of $14.1 \mathrm{MeV}$ also can be used for the direct propulsion. A special design allows neutrons to escape the shield and the blanket of the tokamak. This provides a direct (partial) conversion of the fusion
\end{abstract}


energy into the directed motion of the propellant. In contrast to other fusion concepts, proposed for the space propulsion, this concept utilizes the natural drift motion of charged particles out of the closed magnetic field configuration.

\section{Introduction}

Among all magnetic fusion concepts, the toroidal reactors, such as tokamaks and stellarators, are unique for the fusion space propulsion[1]. They allow a design with the magnetic configuration localized inside the toroidal magnetic field coils, so that propellant ions leaving the ship will not be affected by the magnetic field. In this paper one of the most advanced concepts, tokamak, is investigated as a candidate for the fusion thruster to be used in future deep space missions. While having closed magnetic surfaces for a good plasma confinement and for efficient thermonuclear fusion, the tokamak, as we will show, allows for a "vertical" (along the axis of symmetry) drift motion of plasma energetic charged particles out of the system. Trapped in the ripples of the toroidal magnetic field, these particles at certain conditions can escape the reactor through the gaps between the poloidal coils, which are used in tokamak to create a toroidal field.

In contrast to other fusion concepts, proposed for the space propulsion, this paper, to our knowledge for the first time, utilizes the natural drift motion of charged particles in order to extract plasma ions out of the closed magnetic field system. This directed particle flux can be used as a propellant. By controlling the regime of the tokamak reactor, based on the most realistic deuterium-tritium (D-T) fuel, the whole range of energy between the plasma ion temperature (20-30 keV) up to alpha particle energy of $3.5 \mathrm{MeV}$ can be utilized for the propulsion. As we will illustrate later this has an advantage over the constant high propellant velocity by enhancing the thrust.

Alternative fuels for the fusion reactor promise additional opportunities for the energetic charge particle thruster. In this regard, the deuterium- $H e^{3}\left(D H e^{3}\right)$ fusion, which produces $3.6 \mathrm{MeV} \alpha$-particle and $14.7 \mathrm{MeV}$ proton seems to be interesting for the future advanced reactors. For D-He fusion fuel the energy range may be extended 
up to the proton birth energy. If the flight mission would require to further enhance the thrust one can use the energetic particle flux to heat the plasma confined outside of the closed tokamak toroidal configuration.

Another interesting possibility to extract momentum from the fusion D-T reactor is to utilize second D-T fusion products neutrons, with the energy of $14.1 \mathrm{MeV}$. A special design allows neutrons to escape the shield and the blanket of the tokamak. This creates directed flux of high energy neutrons with the speed of $52000 \mathrm{~km} / \mathrm{sec}$ (which is only $1 / 6$ of the speed of the light). As a result, potentially, the same tokamak reactor on the space craft can cover a wide range of the velocities up to $52000 \mathrm{~km} / \mathrm{sec}$ of propellant particles, which are mostly the by-products of the fusion. This can provide a very efficient direct (and partial) conversion of the fusion energy into the directed motion of the propulsion particles.

Note, that the tokamak is the most developed experimentally and understood theoretically fusion concept, which is on its way to commercial fusion power reactors[1]. Recent fast progress in advanced configurations (e.g., low aspect ratio compact spherical tokamaks), in new regimes and, the most important, in increasing $\beta$ parameter (ratio of the plasma pressure to that of the magnetic field) up to the level of $40 \%$ in average, raises hopes on dramatic reduction in size and weight of the tokamak-reactors[2]. This raises expectations for using advanced tokamak concepts for the space propulsion.

Recently it was proposed that so called low aspect ratio tokamaks, or spherical tori, can be used as a power generator and a source of the plasma[3]. However, as we will see single particle can not leave closed magnetic field configuration with toroidal magnetic field. In this paper we explore the ideas of breaking particle adiabatic moments in the rippled toroidal magnetic field to move particle out of the magnetic configuration for the direct thrust or for the subsequent heating externally confined plasma with lower temperature compare with the tokamak temperature. While the fusion research is concentrated around the plasma confinement, in this paper we are addressing the mechanisms of efficient trapped particle losses for creating the thrust, including losses of charged fusion products (e.g., $\alpha$-particles) and tails of the background ion population.

The purpose of this publication is to establish the concept and to demonstrate its 
theoretical feasibility. The paper is organized as follows. First we are showing the advantages of using tokamak based reactor over the adiabatic traps in Section 2. Then we compare two ways of using the fusion propulsion, i.e. with the constant propellant velocity and with the adjustable velocity in Sec.3. Different geometries of the toroidal magnetic field are studied in Sec.4 to present the proof of principle of the tokamak fusion thruster and to explore the sensitivity of energetic ion extraction on Larmor radius and other plasma parameters. Summary is given in Sec.5.

\section{Why Tokamak, not Adiabatic Traps (Mirror Ma- chines)}

Though many toroidal systems may fit into the concept we propose, the tokamak reactor as a base of the concept is investigated in more details. In simple description the tokamak is represented by the strong toroidal magnetic field created by the poloidal coils. The toroidal field confines the plasma. Single particle in such field moves along the magnetic field lines and performs the gyro motion in the perpendicular to the magnetic field direction. To compensate the perpendicular toroidal magnetic ambipolar drift of the gyro orbit an electric current needs to be generated in the plasma. Toroidal current creates a rotational transform for the particle longitudinal motion so that both electrons and ions are confined (see Ref.[1] for more details). Such configuration is seen as the closest to the actual construction of the fusion reactor. Even though the tokamak concept is the most advanced and closest to the reactor design, its closed magnetic field lines were thought to make it an unusable system for the direct thrust.

On the first glance, among the magnetic fusion concepts the open field line configurations seem to be the most attractive for the fusion propulsion. For example, the magnetic configuration of the mirror-machines (behind the mirror point) look very similar to the nozzle of the jet engine and seems to be ideal to utilize the parallel motion of the plasma particles escaping into the loss-cone for the propulsion. More detailed analysis shows principal problems with the open field mirror machines. Plasma once left the system may come back along the magnetic field lines to another end of the 
trap, so that there is no net thrust. It is almost impossible to neutralize or detach the high temperature plasma from the magnetic field. Special design for the nozzle may be required to solve this problem. Other problem mirror machines face is that the propellant is the plasma itself so that the fuel may be out of the reactor before it is burned out, which will restrict the mass flow of the propellant if we need burning fusion plasma. Tokamaks allow for a design when all its magnetic field is localized inside the toroidal field coils. Only energetic particles can escape such configuration while the fuel is left in the plasma to contribute to the burning. So, if the energetic particle escapes the toroidal field due to the drift motion, there are no obstacles for it to leave the spacecraft. With a statistically preferable direction of motion of such particles, this creates the propulsion momentum for the spacecraft. To further enhance the thrust one could create low temperature plasma outside of the tokamak and use it as a source of the propellant[3]. Energetic particle flux from the tokamak can be used to heat this outside plasma. As we will see at fixed power of the generator the thrust is inverse to the velocity of the propellant, so that at least on the initial stage of the flight one would need such external plasma to boost the space ship.

Adiabatic magnetic traps or mirror machines initially attracted a lot of attention in the magnetic fusion community as candidates for the fusion reactor, but were proved to have a very low energy confinement time, which is critical for building the self sustained burning plasma reactor. Confined plasma inside the machine is left with the so-called loss cone in the velocity phase space. Because of this loss cone, kinetic instabilities prevent the plasma from achieving high energy confinement times as achievable trapping magnetic fields are not strong enough to shrink the loss cone and to achieve the required confinement [4]. In the fusion program it was realized that the mirror machines are far behind the tokamaks in possibility of achieving fusion conditions. This was confirmed also in the recent report at this year APS meeting by the NASA researchers [4]. In the same talk authors proposed more complicated device, which may be interesting but is just an incremental improve of the mirror machines and will face the same problems.

On the other hand, tokamaks were around for a few decades. They are extensively 
studied and are on the verge of being build as a demo reactor[5]. Experimentally already achieved conditions when the power used for plasma heating almost equals to the power released during the plasma discharge[6]. New concepts with high plasma beta and low machine size emerged recently. One of such concepts is known as a spherical tokamak and was studied in US and UK with record achievable beta $\beta_{p} \simeq 40 \%$ (see Ref.[7] and references therein).

Our proposal of the tokamak powered rocket combines the most advanced fusion concept with the direct plasma thrust making the whole concept of fusion powered spaceship extremely attractive, simple and cheap.

\section{Rocket equations and requirements for the propel- lant}

In this section we examine two different approaches to the problem of using the propellant if the power of the generator is fixed. First one is when the velocity of the propellant is fixed. It is compared to the approach of the adjustable propellant velocity. The big advantage of using the controllable propellant velocity is that the thrust can be controlled too.

Let us introduce rocket mass $M$, rocket velocity $V$, mass of the propellant $m$, and its velocity $v$. For now we assume that all propellant particles are leaving the rocket with the same velocity. This allows to solve the problem analytically, while for the practical purposes one would need to use some averaged velocity of the propellant, or a specific thrust[8] and solve the system of differential equations numerically. First, consider Constant Propellant Velocity (CPV) case, which obeys the conventional rocket equation

$$
M(t) \simeq M_{0} \exp \left(-\frac{V(t)}{v}\right),
$$

connecting the spaceship mass at given time $t$ with its mass when it take off at $t=0$. For the consistency we present the derivation of this rocket equation in Appendix A (see also for example Refs [9][8]). For the second scenario, i. e. when the Adjusted 
Propellant Velocity (APV) is evolving according to $v=V / \alpha, \alpha<1$, and the spaceship mass should be determined by the modified rocket equation (see Appendix A)

$$
M(t) \simeq M_{0}\left(\frac{V_{0}}{V}\right)^{\alpha}
$$

Consider the tokamak based generator producing $P$ gigawatt power. Note, that planned ITER prototype of the tokamak-reactor is designed for $P \simeq 1 G W[5]$. We also assume that the power is directly transformed into the thrust with the efficiency $\eta$ less then one, which gives the fraction of power utilized for heating of the propellant. The amount of thrust produced by the propellant is given by Eq.(22) and can be expressed in terms of generated power

$$
F=2 \eta P / v \text {. }
$$

From this equation one can see that APV offers more flexibility to optimize the use of generated power, the lower $v$ the higher thrust is. Obvious requirements for the space flight is to minimize both ratio $M_{0} / M>1$ and flight time.

In APV case, Eq.(2), one needs finite initial velocity $V_{0}$ to get finite $M_{0}$. Therefor to boost the rocket to that speed, CPV scheme with small $v$ can be used. This follows also from the requirements that the propellant may have some minimum velocity $v_{\text {min }}$. Assume also that at this stage, denoted below by the subscript 1, with maximum thrust (see $\mathrm{Eq}(3)$ ) the spaceship velocity is limited by the inequality $V<v_{\min } \alpha$. The equation for $V(t)$ is then

$$
\frac{d V}{d t}=\frac{2 \eta P}{v_{\min } M_{0}} e^{\left(V-V_{0}\right) / v_{\min }}
$$

and has a solution

$$
V-V_{0}=-v_{\min } \ln \left[-\frac{2 \eta P}{v_{\min }^{2} M_{0}}\left(t-t_{0}\right)+1\right],
$$

which implies that the time required to speed up the rocket to $V_{1}=v_{\min } \alpha$ is

$$
t_{1}-t_{0}=\frac{v_{\min }^{2} M_{0}}{2 \eta P}\left[1-e^{-\left(V_{1}-V_{0}\right) / v_{\min }}\right] \simeq \frac{v_{\min }^{2} M_{0} \alpha}{2 \eta P}
$$


On a second stage, APV, i.e. $V=\alpha v$, we make use of Eq.(2) with the substitution $0 \rightarrow 1$. Evolution of $V$ is described by

$$
\frac{d V}{d t}=\frac{2 \eta P \alpha}{M_{1} V_{1}^{\alpha}} V^{\alpha-1}
$$

and has a solution

$$
V=\left[V_{1}^{2-\alpha}+\frac{2 \alpha(2-\alpha) \eta P}{V_{1}^{2} M_{1}}\left(t-t_{1}\right)\right]^{1 /(2-\alpha)}
$$

It will take

$$
t_{2}-t_{1}=\left[\left(\frac{V_{2}}{V_{1}}\right)^{2-\alpha}-1\right] \frac{V_{1}^{2} M_{1}}{2 \eta P \alpha(2-\alpha)},
$$

to accelerate the spaceship up to speed $V_{2}=\alpha v_{\max }$, which is the limit for the second stage, where $v_{\max }$ is the limit velocity for the propellant equal to the birth velocity of fusion products. Therefore, the third and final stage is again CPV and has to follow Eqs. $(5,6)$ with the substitution $0 \rightarrow 2$ and $1 \rightarrow 3$. On stage 3 the spaceship will be accelerated until it reaches the designated velocity. Depending on the mission the ship might reach necessary velocity at stage one or two. Below we will refer with APV notation for the combined 3 stage scenario CPV-APV-CPV.

To illustrate the effectiveness of adjustable propellant velocity we introduce dimensionless variables

$$
\tau=\frac{2 \eta P t}{v_{\min }^{2} M_{0}}, u=\frac{V}{v_{\min }} .
$$

The solution for the whole flight can be written in the form

$$
u=\left\{\begin{array}{cc}
-\ln (-\tau+1), & \tau<\tau_{1} \\
{\left[\alpha^{2-\alpha}+\frac{(2-\alpha)}{\alpha^{\alpha-1} e^{-\alpha}}\left(\tau-\tau_{1}\right)\right]^{1 /(2-\alpha)},} & \tau_{1}<\tau<\tau_{2}, \\
u_{2}-\frac{v_{\max }}{v_{\min }} \ln \left[\left(\tau_{2}-\tau\right)\left(\frac{v_{\min }}{v_{\max }}\right)^{2-\alpha}+1\right], & \tau_{2}<\tau<\tau_{3}
\end{array}\right.
$$

where $\tau_{1}=1-e^{-\alpha}, u_{2}=\alpha v_{\max } / v_{\min }, \tau_{2}=\tau_{1}+\alpha e^{-\alpha}(2-\alpha)^{-1}\left[\left(v_{\max } / v_{\min }\right)^{2-\alpha}-1\right]$, 
$u_{3}=V_{3} / v_{\min }$ is the final rocket velocity. The final spaceship mass will be connected with its mass at the start according to

$$
M=\left\{\begin{array}{cc}
M_{0} e^{-u}, & \tau<\tau_{1} \\
M_{0} e^{-\alpha}\left(\frac{\alpha}{u}\right)^{\alpha}, & \tau_{1}<\tau<\tau_{2}, \\
M_{0} e^{-u v_{\min } / v_{\max }}\left(\frac{v_{\min }}{v_{\max }}\right)^{\alpha}, & \tau_{2}<\tau<\tau_{3}
\end{array}\right.
$$

We compare this approach with the conventional rocket equation of fixed propellant velocity $v=v_{\max }$, in which spaceship velocity is determined by

$$
u=-\frac{v_{\max }}{v_{\min }} \ln \left[-\tau \frac{M_{0}}{M_{0}^{\prime}}\left(\frac{v_{\min }}{v_{\max }}\right)^{2}+1\right],
$$

where the prime will refer to the case with CPV, $M_{0}^{\prime}$ is initial mass of the rocket calculated in such a way that at $\tau_{3}$ the final mass, $M_{f}$, is the same for both cases, CPV and APV,

$$
M_{0}^{\prime}=M_{f}+\tau_{3}\left(\frac{v_{\min }}{v_{\max }}\right)^{2}
$$

Figures 1, 2, 3

present a comparison of the spaceship velocity evolution for two propellant velocity time dependencies, APV and CPV. One can see that the APV offers the fastest way to achieve the final velocity $u_{3}$ but for the price of larger starting mass. On the other hand using maximum propellant velocity gives a strong advantage by reducing the mass of the spacecraft.

To estimate the required rocket mass for different missions one need to integrate the above velocity expressions $(11,13)$ to calculate the flight path time dependence. 


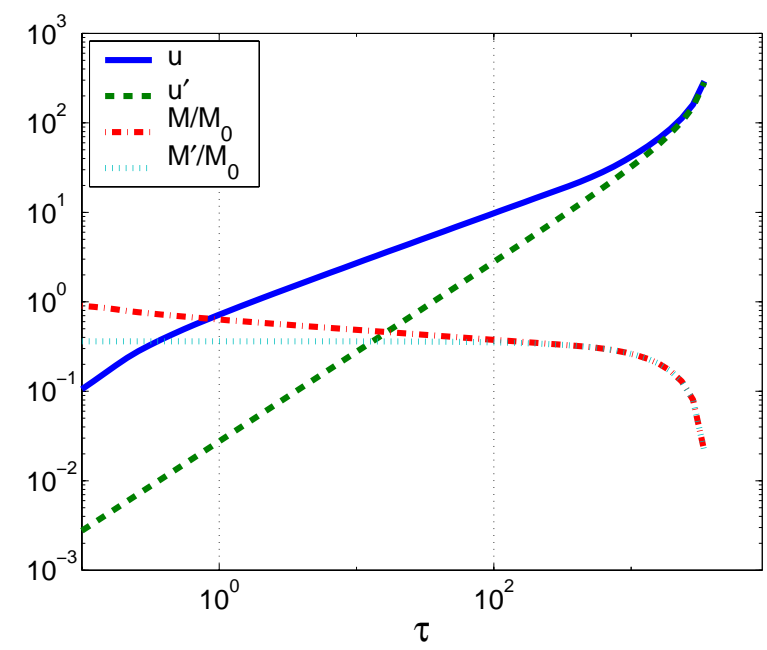

Figure 1: Comparison of the performance of APV solution, Eq.(11), with the simple rocket equation solution, CPV, Eq.(13) at $\alpha=1 / 5$, where stages are separated by times $\tau_{1}=0.18, \tau_{2}=362, \tau_{3}=9.6 \tau_{2}$.

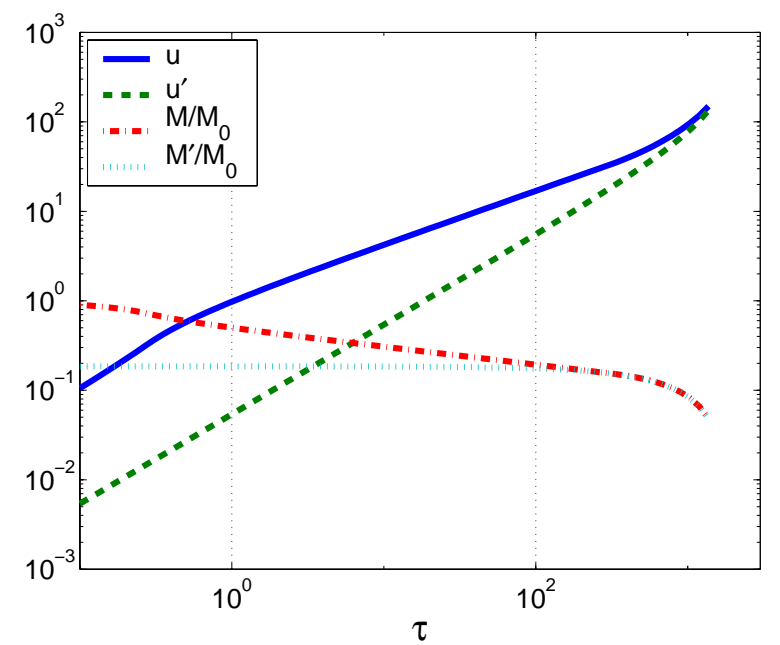

Figure 2: The same as Fig.1 but for $\alpha=1 / 3, \tau_{1}=0.28, \tau_{2}=309, \tau_{3}=5.5 \tau_{2}$. 


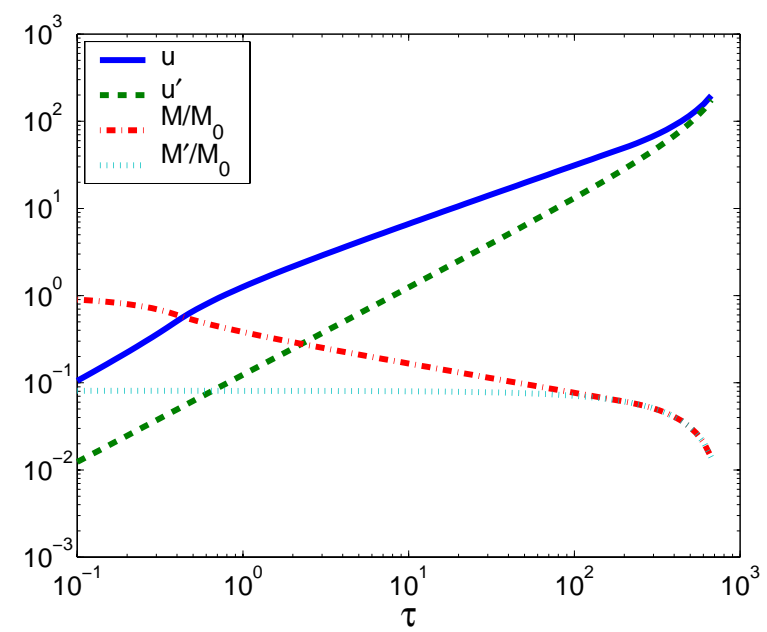

Figure 3: The same as Fig.1 but for $\alpha=1 / 2, \tau_{1}=0.39, \tau_{2}=202, \tau_{3}=3.5 \tau_{2}$.

The result for APV is

$$
l(t)=\left\{\begin{array}{ll}
(1-\tau) \ln (-\tau+1)+\tau, & \tau<\tau_{1} \\
l\left(\tau_{1}\right)+\frac{\alpha^{2}}{(3-\alpha) e^{\alpha}}\left\{\left[1+\frac{2-\alpha}{\alpha} e^{\alpha}\left(\tau-\tau_{1}\right)\right]^{\frac{3-\alpha}{2-\alpha}}-1\right\}, & \tau_{1}<\tau<\tau_{2} \\
l\left(\tau_{2}\right)+u_{2}\left(\tau-\tau_{2}\right)+\frac{v_{\max }}{v_{\min }}\left\{\tau-\tau_{2}+\left[\left(\frac{v_{\max }}{v_{\min }}\right)^{2-\alpha}-\tau+\tau_{2}\right] \times\right. & \\
\left.\times \ln \left[\left(\tau_{2}-\tau\right)\left(\frac{v_{\min }}{v_{\max }}\right)^{2-\alpha}+1\right]\right\} & \tau_{2}<\tau<\tau_{3}
\end{array} .\right.
$$

The conventional rocket equation solution, CPV, corresponds to the path:

$$
l^{\prime}(t)=\frac{v_{\max }}{v_{\min }}\left\{\tau+\left[\frac{M_{0}^{\prime}}{M_{0}}\left(\frac{v_{\max }}{v_{\min }}\right)^{2}-\tau\right] \ln \left[1-\tau \frac{M_{0}}{M_{0}^{\prime}}\left(\frac{v_{\min }}{v_{\max }}\right)^{2}\right]\right\} .
$$

Figures 4, 5, 6

give a comparison of the overall performance of CPV and APV flight scenarios. Obtained expressions are general and will be used here to evaluate the performance of fusion rocket basing on reasonable assumptions of the key parameters of fusion spacecraft. For the simplicity we take D-T alpha particle birth velocity as maximum propellant velocity $v_{\max }=1.3 \times 10^{9} \mathrm{~cm} / \mathrm{sec}$ and $v_{\max } / v_{\min }=100$, which corresponds to 


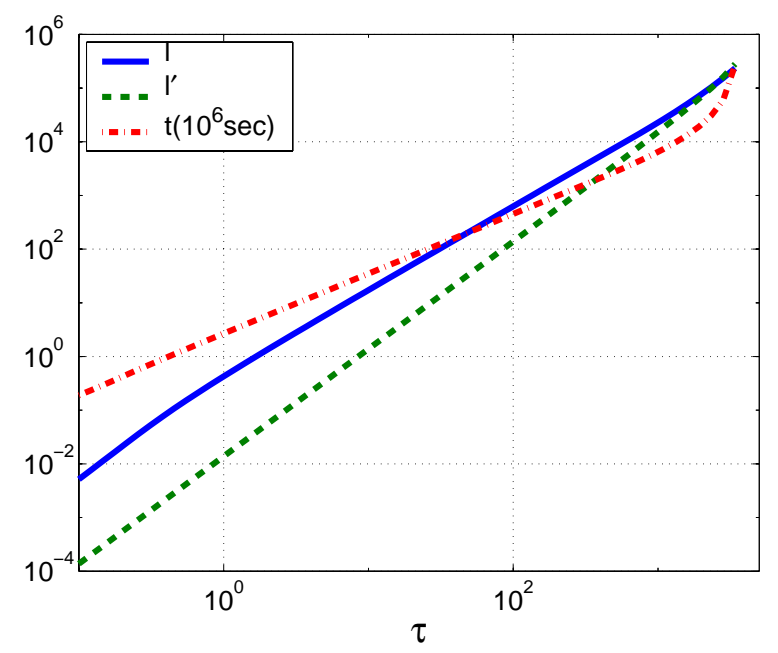

Figure 4: Normalized flight path vs. time dependence for the same spaceship parameters as in Fig.1.

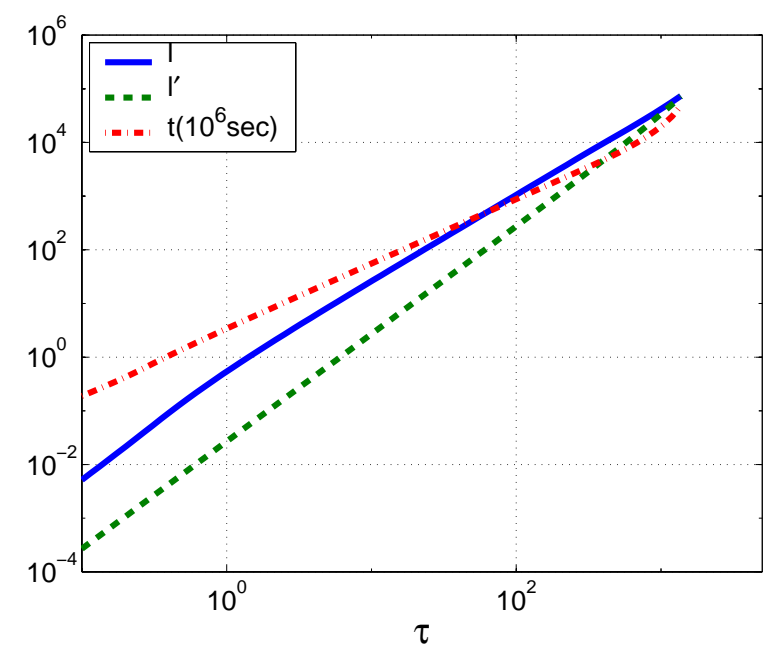

Figure 5: Normalized flight path vs. time dependence for the same spaceship parameters as in Fig.2. 


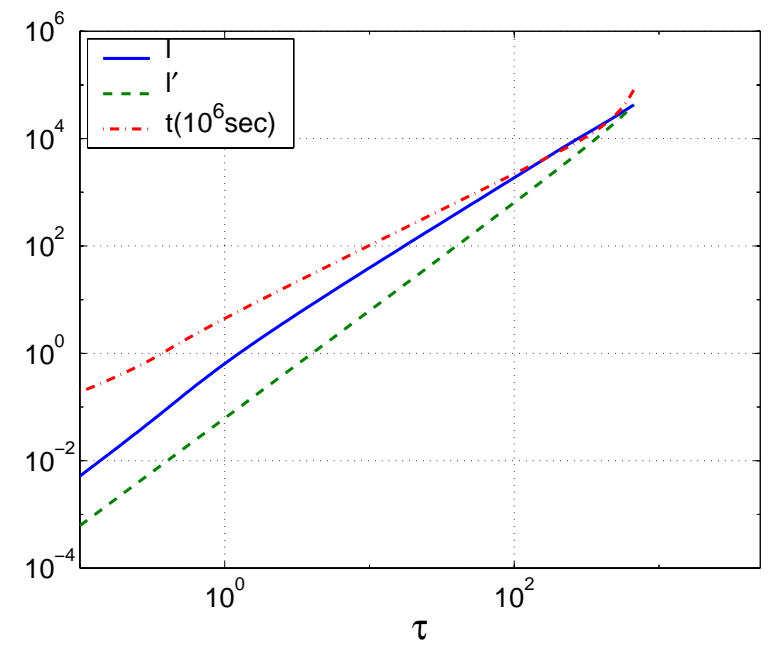

Figure 6: Normalized flight path vs. time dependence for the same spaceship parameters as in Fig.3.

thermal velocity of the deuterium plasma used for the propellant with the temperature $T_{\min }=m_{D} v_{\min }^{2} / 2=0.2 \mathrm{keV}$. The weight of the rocket is not easy to estimate, since the tokamak reactor is far from being ready for the power plant. To start with we will use the power and the size of the ITER design [5] with the weight of the spaceship mainly consisting of $1 \mathrm{~m}$ thick blanket with the mass density of water, which is, recall, equal to double mass density of the lithium. Note, that liquid lithium is being recently considered as a material for the blanket and first wall covering [14]. The major radius of ITER plasma is $R_{0}=6 \mathrm{~m}$, and minor radius is $a=2 \mathrm{~m}$ so that the net estimate for the weight of the rocket including the power generator is on the order of 100tons.

It is easy now to find a required time for the flight using the length and time normalization constants $v_{\min }^{3} M_{0} / 2 \eta P=2.2 \times 10^{8} \mathrm{~km}$ and $v_{\min }^{2} M_{0} / 2 \eta P=1.7 \times 10^{6} \mathrm{sec}$, where we assumed $\eta=0.5$. Four curves, shown in Fig. 7 correspond to APV model with three values of parameter $\alpha$ and the conventional CPV model. Normalized values of $l$ and $\tau$, as well as mass ratios, can be found from the figures 4, 5, 6, where the real time $t, s e c$ is shown. Since $L$ curves are very close to each other we have separated them on Fig. 7 by multiplying $\alpha=1 / 3$ and $\alpha=1 / 5$ curves by 10 and 100 , respectively.

One can notice that there is almost an order of magnitude advantage in time for a 


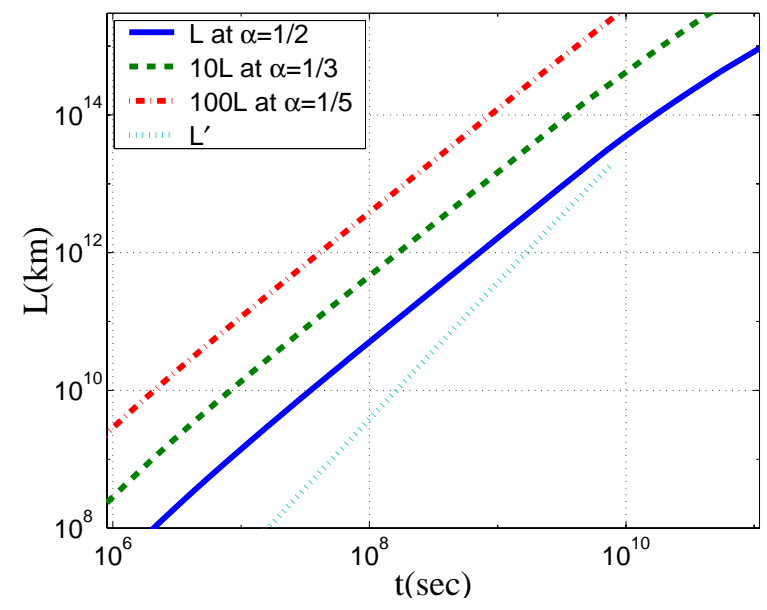

Figure 7: Real distance from the starting point versus time for the space flight according to the modified rocket equation, APV, and conventional rocket equation, CPV.

given distance of APV curve dependence over the CPV curve dependence. According to Fig. 7 it will take around 1 year to rich the Pluto. i.e. $L=10^{10} \mathrm{~km}$. Chosen parameters also require around 300 years to go to the closest star Proxima Centauri, which is $\sim 0.4 \times 10^{14} \mathrm{~km}$ away from the earth. Better performance of the spaceship can be expected if we improve parameters so that $v_{\max }=5.2 \times 10^{9} \mathrm{~cm} / \mathrm{sec}, v_{\max } / v_{\min }=400$ and the power of the fusion reactor $P=10 G W$. Note that the case with similar parameters was discussed in Ref.[3]. In this case as shown in Fig. 8 the required time is reduced to about 100 years. Such velocity $v_{\max }$ have fusion protons in $\mathrm{D}-\mathrm{He}^{3}$ reaction. Further 3 time reduction in the flight time can be achieved if the power to mass ratio is increased one more order of magnitude. This is possible in principle, by constructing bigger machine since the power of the tokamak reactor increases as a volume, i.e. $P \sim R_{0}^{3}$, while the mass increases as a surface area $M \sim R_{0}^{2}$. Detailed analysis of such reactor is out of scope of this paper.

In the above estimates we did not account for the spaceship slowing down portion of its trajectory and part of the flight when the thrust is used against the gravitational field. These contributions to the required time is smaller than ones calculated, since in our analysis we fixed the generated power, so that during the slowing down phase 


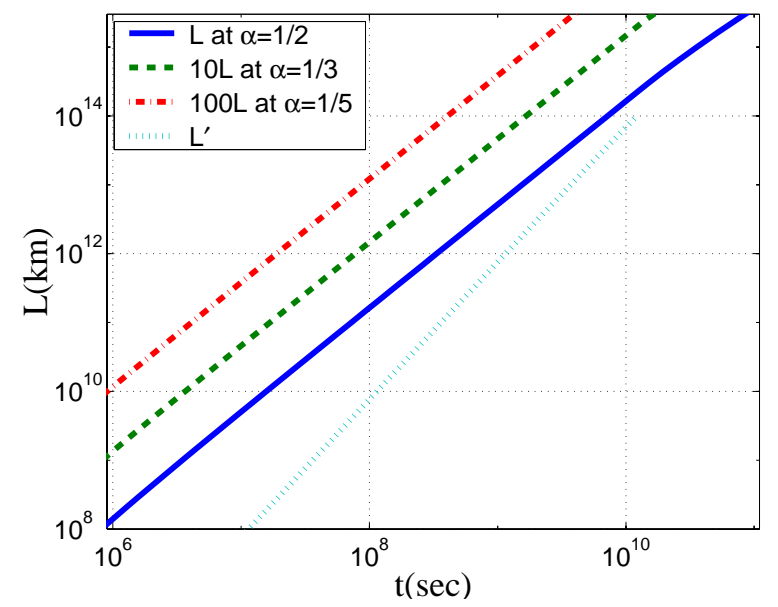

Figure 8: The same as in Fig.7 but for $v_{\max } / v_{\min }=400$ and $P=10 G W$.

at the end of the trip the mass of the ship is smallest and the ship acceleration is the largest (see Eq.(3)).

We have to note again, that in the derivation the propellant velocity should be substituted with the specific thrust which is an average velocity per mass flow of the propellant. The exact problem needs numerical solution where the propellant should have a velocity distribution.

Nevertheless, the solution to the problem of deep space propulsion we propose has obvious advantage of using the APV concept. Expressions we obtained can be readily used when developing the concept once the tokamak reactor design becomes more practical. At this point we can argue from the analysis above that there is a theoretical feasibility of using the fusion tokamak based spaceship for the interplanetary and interstellar missions. For now we consider other critical problem of extracting the directed particle flux from the tokamaks. 


\section{Energetic ion extraction from the tokamak mag- netic configuration via the magnetic field ripple loss mechanism}

In this section we explore the extraction of ions from the closed field line magnetic configuration in details, since it is a key to the concept of toroidal fusion propulsion. Our idea of using ripple diffusion is new and distinguish our toroidal fusion concept from other concepts to use tokamak or other fusion devices for the propulsion.

In the toroidal magnetic configurations under the consideration, the charged particle is drifting along the axis of symmetry due to the gradient of the toroidal magnetic field. The direction of the drift depends on the sign of the particle charge. In reality the toroidal magnetic field is not perfectly axisymmetric. Due to the finite distance between the toroidal field coils magnetic field has oscillations in absolute value along the field line, i.e. so called ripples. As a result without the plasma magnetic field is a periodic function of the distance along its line and has the period equal to the distance between the coils.

It is well known that in tokamaks due to ripples charged particle can stochastically diffuse in the direction of the drift, get trapped in the magnetic well between the toroidal field coils, and eventually be lost. However only energetic particles are effected by this mechanism which may produce both indirect and direct thrust. Stochastic ripple diffusion in tokamaks has threshold as was shown in Ref.[11]

$$
\delta>\delta_{c r}=\rho_{L}^{-1} q^{-1}(\pi N q / \epsilon)^{-3 / 2}
$$

where $\delta=\left(B_{\max }-B_{\min }\right) /\left(B_{\max }+B_{\min }\right)$ is the magnitude of the magnetic field ripple, $B_{\max }$ and $B_{\min }$ are the maximum and minimum of the magnetic field along the field line in the tokamak vacuum magnetic configuration, $\rho_{L}=v_{\perp} / \omega_{c}$ is particle Larmor radius, $v_{\perp}$ is particle perpendicular velocity, $\omega_{C}$ is the cyclotron frequency, $\epsilon=a / R$ is the tokamak inversed aspect ratio, $q=B_{\theta} r / B_{\varphi} R$ is the, so called, safety factor, $r$ is the plasma minor radius, $B_{\theta}$ and $B_{\varphi}$ are the poloidal and toroidal magnetic field 


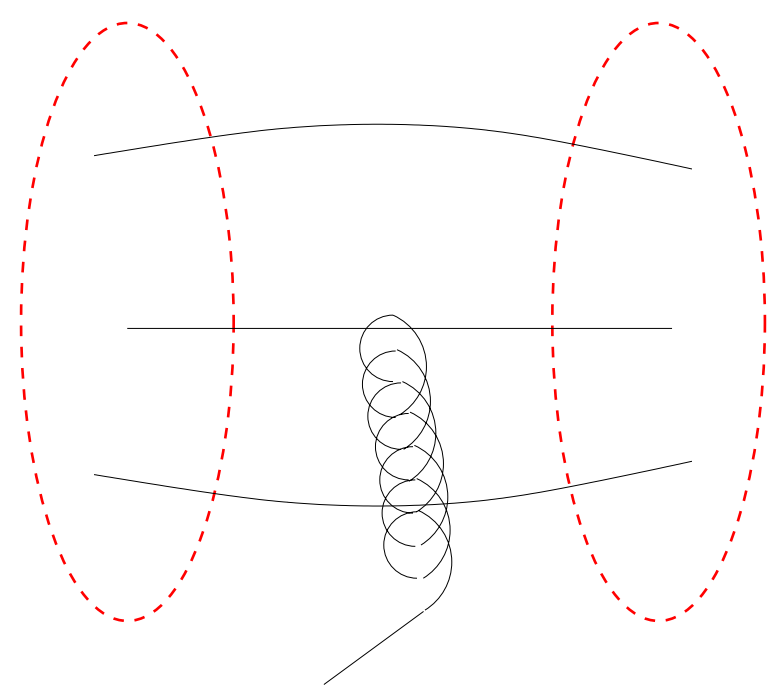

Figure 9: The sketch of the charged particle orbit when it becomes trapped between the toroidal field coils. Shown also are two neighboring coils.

components, respectively. The quantity $\delta$ is typically an exponential function of $r$ increasing rapidly from the center to the edge, so that the effect of ripples is very selective in space. The following Figure 9 gives a sketch of particle motion if it becomes trapped between the toroidal field coils.

The magnitude of ripples depends on the design of the toroidal field coils, in particular, their size, number of coils and the structure of the current distribution in the coils. This dependence can be used for the control of the thrust. For this purpose, the toroidal field coils can be designed in such a way, that the asymmetric harmonics of the current distribution, responsible for the ripple amplitude, can be controlled independently from the total current in the coils.

In the plasma, only trapped particles are affected by this loss mechanism. The amount of trapped particle population is equal to $\sqrt{2 \epsilon}$ of the total number of particles for isotropic particle distribution. It means that only a fraction of energetic particles, such as charged fusion products will be effected. The rest of energetic particles will contribute to the energy balance of the burning fusion plasma. In order to create more thrust one can use the Ion Cyclotron Resonance Heating (ICRH) of charged 
particles, the technique widely used in the tokamak research[1]. ICRH is efficient in scattering particles from the passing to the trapped domain in the velocity phase space, where particles will be affected by the ripples. With this process even background plasma Maxwellian tail ions will be forced to leave the reactor and create thrust as was demonstrated experimentally in ICRH plasma discharges in many tokamaks [10].

\subsection{Coil design requirements}

Once being trapped in the toroidal field well particle starts to move along the lines of constant magnetic field with the drift velocity to the zero order in $\rho_{L} / R_{0}$ [12]:

$$
v_{d r}=\frac{\left(v_{\|}^{2}+v_{\perp}^{2} / 2\right)}{\omega_{c}} \mathbf{b} \times \nabla \ln B
$$

where $\mathbf{b}=\mathbf{B} / B$. This means that single particle having small Larmor radius can never leave tokamak magnetic configuration. Plasma particles are becoming trapped on the low field side of the tokamak, where the ripples are strongest. Then moving along the $B=$ const lines drifting particle approaches the outer boundary of the toroidal magnetic field coil on the High Field Side (HFS) and returns back to Low Field Side (LFS). To allow particle to leave the closed configuration one needs to exploit the nonconservation of $\mu$. If coils are designed in such a way, that the distance from the particle trajectory to the outer boundary of the coils becomes comparable to the Larmor radius of the particle, it can leave the toroidal configuration. Thus, the coil radial size should be larger then particle Larmor radius at the low field side $\Delta r_{L F S} \gg \rho_{L 0}\left(1+a / R_{0}\right)$. Particle moves on the line $B \simeq B_{0} /\left(1+a / R_{0}\right)$ and to leave the configuration it should approach the outer boundary of HFS part of toroidal field coil within the distance smaller then the Larmor radius $\Delta r_{H F S}\left(1-a / R_{0}\right) /\left(1+a / R_{0}\right) \ll \rho_{L 0}\left(1+a / R_{0}\right)$, where we assumed that the magnetic field changes linearly across the coil. Thus the coil should have radial dimensions satisfying the following conditions

$$
\Delta r_{L F S} \gg \rho_{L 0}\left(1+a / R_{0}\right), \Delta r_{H F S} \ll \rho_{L 0}\left(1+a / R_{0}\right)^{2} /\left(1-a / R_{0}\right) .
$$




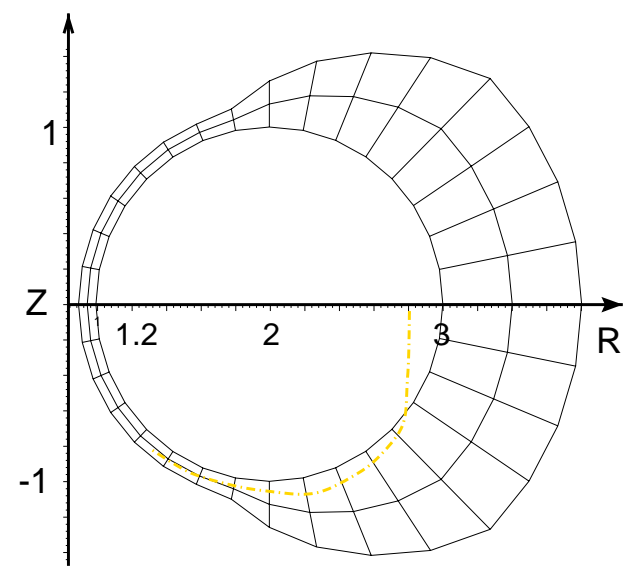

Figure 10: Toroidal field coil for one of the configuration studied below with major radius $R_{0}=2$, and minor radius $r=1$. Shown also a sketch of particle guiding center orbit below the midplane.

This special coil design meeting these criteria is illustrated on next figure 10 with the sketch of the part of particle guiding orbit trajectory. Below this concept will be included into the calculations.

\subsection{Magnetic field model}

We developed numerical codes to simulate the energetic particle behavior in the realistic rippled magnetic field of the tokamak. The magnetic field was calculated with a given toroidal coil geometry (see Fig.10) and splined into 3D splines to enhance the numerical performance so that the resulting magnetic field vector is a function of three spatial coordinates determined by the spline coefficients at the point of interest

$$
\mathbf{B}=\mathbf{B}(R, Z, \varphi)
$$

Such approach allows efficiently calculate vacuum magnetic field for the particle equations of motion.

To describe the equilibrium state of the plasma magnetic field the high aspect ratio approximation $(\epsilon \rightarrow 0)$ with homogeneous current density ( $j_{0}=$ const) was used[13]. 
The radial dependence of the magnetic field is taken in the form

$$
B(\rho)=\left\{\begin{array}{c}
\frac{C I_{0} \rho}{a^{2}}, 0 \leq r \leq a \\
\frac{C I_{0}}{r}, r>a
\end{array}\right.
$$

where $I_{0}$ is total plasma current. The equation for the poloidal magnetic flux is given by the solution $\psi(r)=\int_{0}^{r} 2 \pi R B d r$ keeping equal left and right derivatives at the edge $\psi_{\mid r=a} \prime(r \leq a)=\psi_{\mid r=a} l(r \geq a)$. Thus, the expression for the poloidal magnetic flux can be obtained as follows

$$
\psi(r)=\frac{2 \pi B_{t a} R_{a} a^{2}}{q_{a} R_{0}}\left\{\begin{array}{c}
\left(\frac{r}{a}\right)^{2}, 0 \leq r \leq a \\
\left(1+2 \ln \frac{r}{a}\right), r>a
\end{array}\right.
$$

where $B_{t a}$ is the toroidal magnetic field, $q_{a}$ is the safety factor at the plasma edge, $R_{a}=$ $R(a)$. Here, to determine the factor $C I_{0}$, two expressions for the poloidal component of the magnetic field taken at the plasma edge were used

$$
B_{p o l}(a)=\left\{\begin{array}{c}
\left.\frac{B_{t} \rho}{q(\rho) R_{0}}\right|_{\rho=a} \\
\left.\frac{\psi \prime}{2 \pi R}\right|_{\rho=a}
\end{array} .\right.
$$

Components of magnetic field in spatial coordinates are given by the following formula

$$
\begin{aligned}
& B_{R}=\frac{1}{2 \pi R} \frac{\partial \psi}{\partial Z}=\frac{1}{2 \pi R} \frac{\partial \psi}{\partial r} \frac{\partial r}{\partial Z} \\
& B_{Z}=\frac{-1}{2 \pi R} \frac{\partial \psi}{\partial R}=\frac{-1}{2 \pi R} \frac{\partial \psi}{\partial r} \frac{\partial r}{\partial R}
\end{aligned} .
$$

The variable $r$ is expressed in spatial coordinates by

$$
r(R, Z)=\left(8 R\left[3 R+R_{a x}-4\left(R^{2}-R\left(R-R_{a x}\right) / 2-\left(Z-Z_{0}\right)^{2} / 16\right)^{1 / 2}\right]\right)^{1 / 2}
$$

Individual particle obeys following dimensionless equation of motion:

$$
\frac{d \mathbf{v}}{d t}=\omega_{c}[\mathbf{v} \times \mathbf{b}]
$$

where $\mathbf{v}$ is velocity vector of particle. 
To demonstrate the particle extraction in the tokamak we consider several configurations of the plasma keeping edge value of $q$ fixed. We will use dimensionless parameters below. We choose minor radius $a=1$ and three major radii $R_{0}=1.2,2, \& 3$, so that the aspect ratio changes respectively. We also change the number of toroidal coils from 5, 10 to 20.

The magnetic field coils are designed to satisfy Eq.(17) as follows. The LFS width is chosen to be 1 , while the HFS is 0.1 . The coil radial width is slowly changing from the LFS to HFS. Each coil is represented by three wires with one going on the outer boundary, second going on the inner boundary, and third going in between (see Fig.10 for $R_{0}=2$ ).

In calculations we start with the homogeneous particle distribution over the plasma cross section, so that our estimates will be on the low side of the ion extracting performance. The is because for example particle birth profile is peaked at the center of the plasma. In the numerical run all particles have one of the following the Larmor radius to major radius ratios $\rho_{L} / R_{0}=0.04,0.08,0.16, \& 0.32$. Particles are also homogeneously distributed over the Larmor radius phase with 6 different initial phase angles. Toroidally particles are launched between the coils, which is the most probable case for the particle trajectory to end up after being trapped in the ripple. Particle pitch angle is taken zero at the starting point $v_{\|} / v=0$, which corresponds to the bounce point on particle trajectory[1]. This assumption is not important for the answer to the whole problem in the realistic geometry with the plasma current, since particle change its pitch angle and process toroidally. When particle leaves the system we record the moment it carries along the vertical axis $p_{z}$. Each particle can carry maximum moment $p_{z}=1$. At the end of the numerical run we calculate the average moment of particles carried away at given toroidal configuration

$$
P_{z}=\sum_{j} p_{z j} / N
$$

where $N$ is the number of particles. 

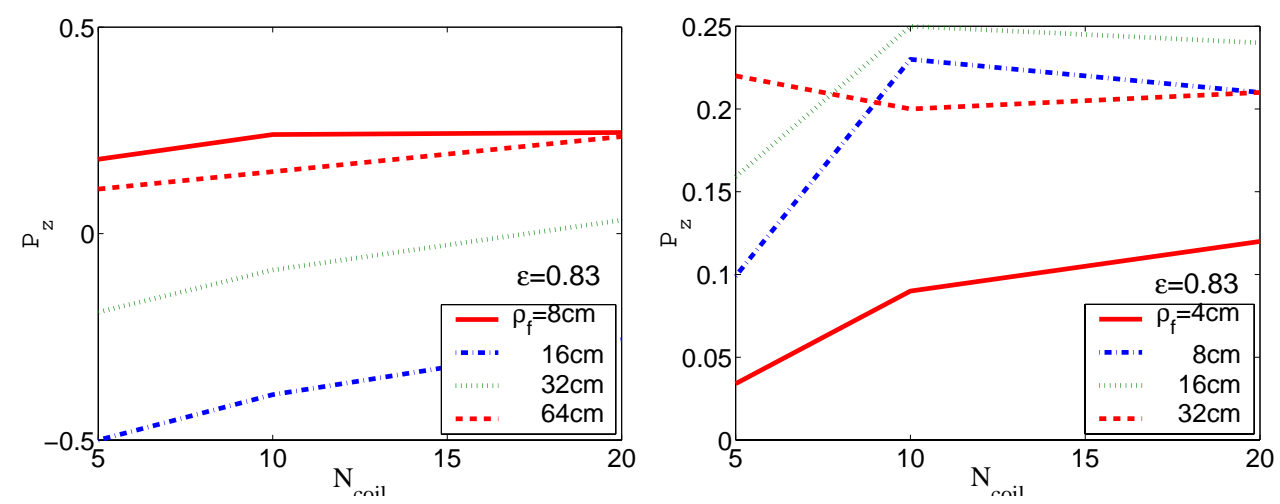

Figure 11: Averaged particle vertical momentum depends strongly on the number of toroidal field coils, shown at fixed inversed aspect ratio $\epsilon=0.83$ for the case with no plasma (left) and with the model equilibrium, Sec. 4.2 (right).

\subsection{Numerical modeling of ripple induced fast ion extraction}

Figure 11 shows the comparison of the results for the vacuum toroidal magnetic field and for the configuration with the toroidal plasma current as described in the previous subsection Eq.(18). Particles carry larger momentum when the plasma current is included as one can see from the Fig. (11 right) and Fig. 12. The most promising configuration has moderate aspect ratio with $\epsilon=0.5$ (Fig.12). The dependence is more smooth for this case as a functions of Larmor radius, which means that more particles will be contributing to the flux.

The space distribution of the Larmor phase averaged momentum in the poloidal cross section of the tokamak is shown in Fig. (13). In the case with the current particle losses are distributed almost over the all poloidal cross section with some fluctuations, which may be due to the numerical errors. Since in our calculations we did not specify any particle birth profile, more realistic profiles can lead to the enhanced particle fluxes. However such an optimization is beyond the scope of this paper.

Very important issue is how particles are distributed in the toroidal angle after they are lost. Fig. (14) gives such a distribution for the case $N_{\text {coil }}=20, \epsilon=0.5$, $\rho_{L} / R_{0}=0.08$. At the left and right limits of x-axes toroidal field coils are located. The distribution was taken $1 \mathrm{~m}$ above or below the coils. This give some averaging of 

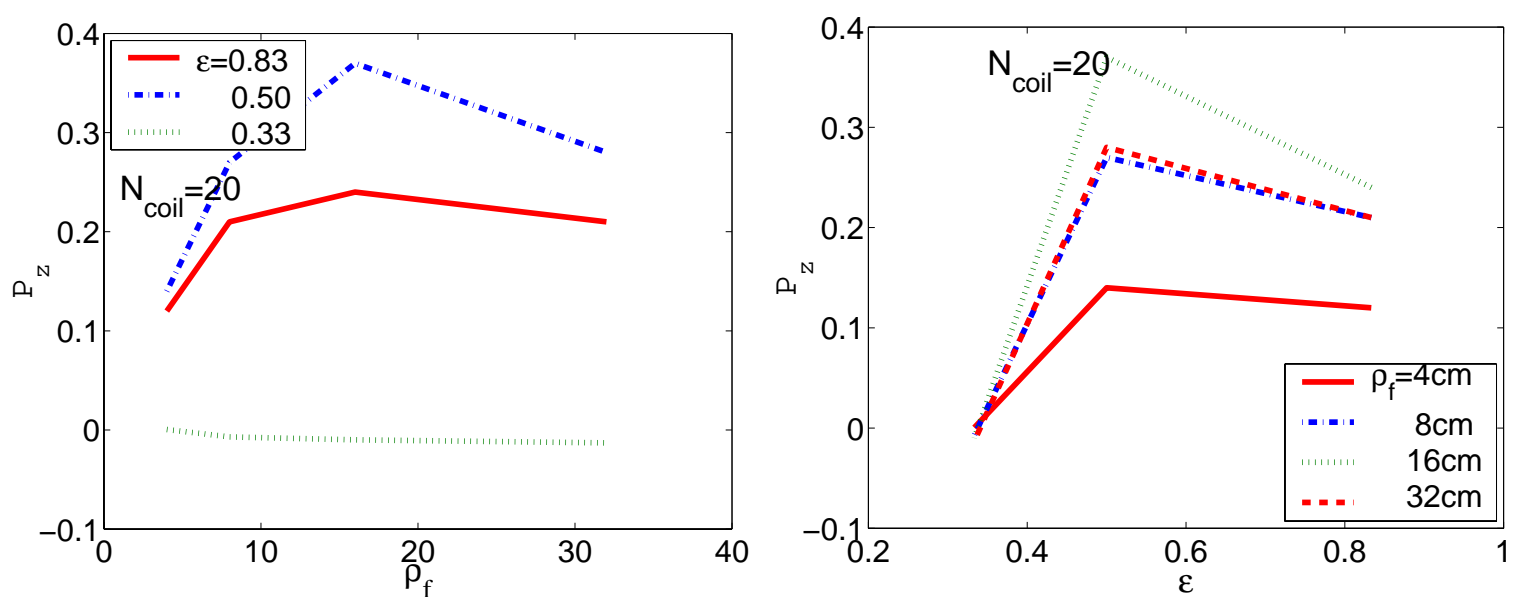

Figure 12: Averaged vertical momentum of particles for different aspect ratios and particle Larmor radii presented as functions of particle Larmor radius (left) and inversed aspect ratio (right) at fixed number of toroidal coils $N_{\text {coil }}=20$.
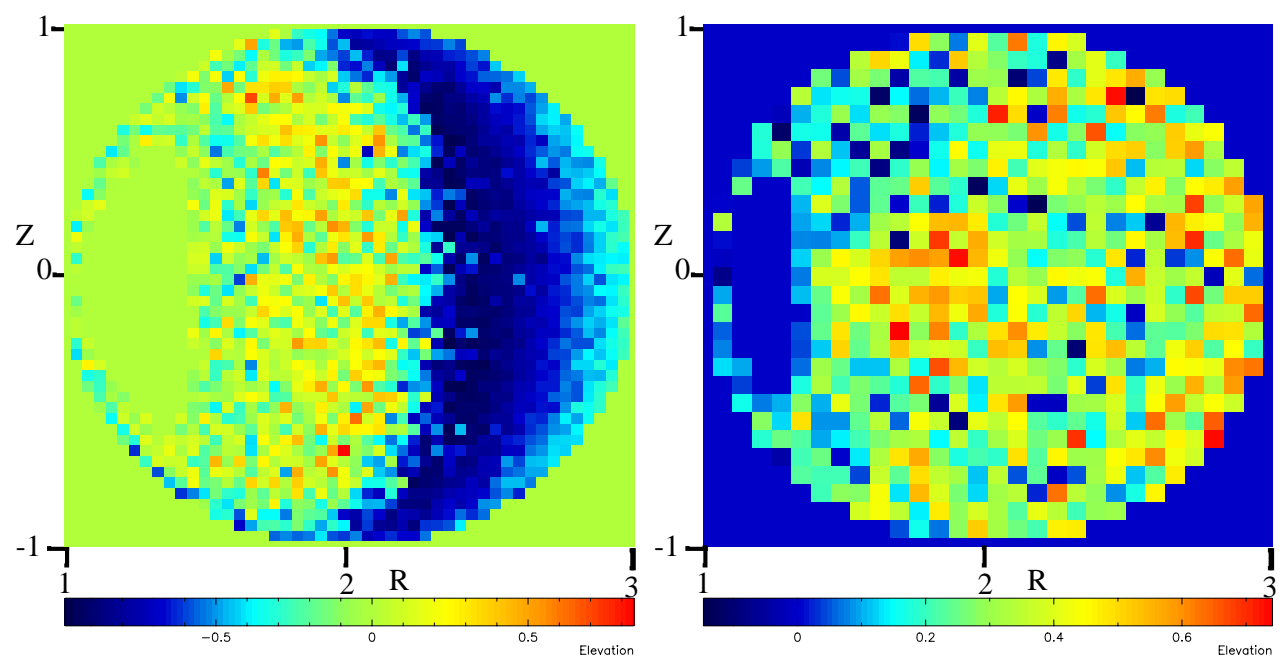

Figure 13: Contours of the vertical momentum carried by particles, which are born at $R, Z$ point. Left figure corresponds to the vacuum toroidal field, and the right figure corresponds to the plasma with the current. 


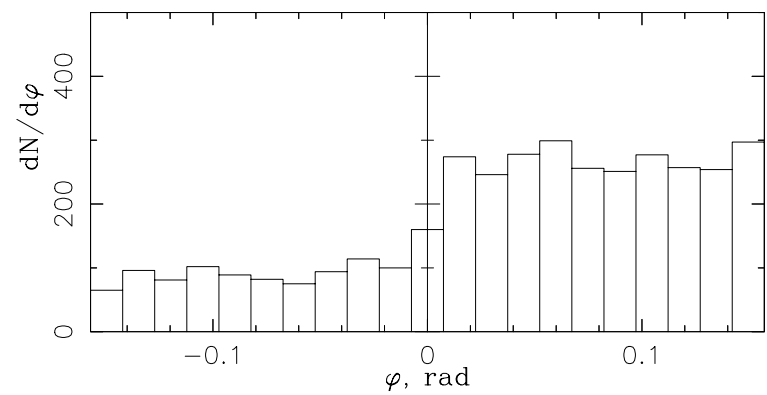

Figure 14: Toroidal angle distribution of particle losses for the case with $N_{\text {coil }}=20$, $\epsilon=0.5, \rho_{L} / R_{0}=.08$.

distribution in comparison with the one near the coil, but still one can see that the majority of ions are lost between the coils. There will be finite number of particles, which could potentially interact with the coils causing their damage. Principal solution to this is to make coils at the parts, where they are most probably will interact as a wires, just like we have in the code, Fig.10. In such a case strong magnetic field near the coil can deviate particle and be untouched.

We have demonstrated numerically that superthermal particles can be extracted from the tokamak with the statistically dominant direction of their velocity. This is the basis for the developing the fusion propulsion spaceship powered by the tokamakreactor.

\subsection{Direct neutron thrust.}

At present, the D-T fusion reactors are the most realistic for the near future. In this case fusion $14 \mathrm{MeV}$ neutrons can be utilized for the thrust. This can be done by placing the blanket, such as liquid lithium, on the upper half of the tokamak-reactor as shown in Fig.15. This blanket has to be placed around the fusion reactor to absorb the energy carried by neutrons. It needs to be at least a few tens of centimeters thick to absorb the majority of neutrons. Half the neutrons can be used for the thrust while other half will be used for the energy generation and partial tritium recycling. Only part of particle momentum is used, so that the efficient velocity of neutron propellant can be 


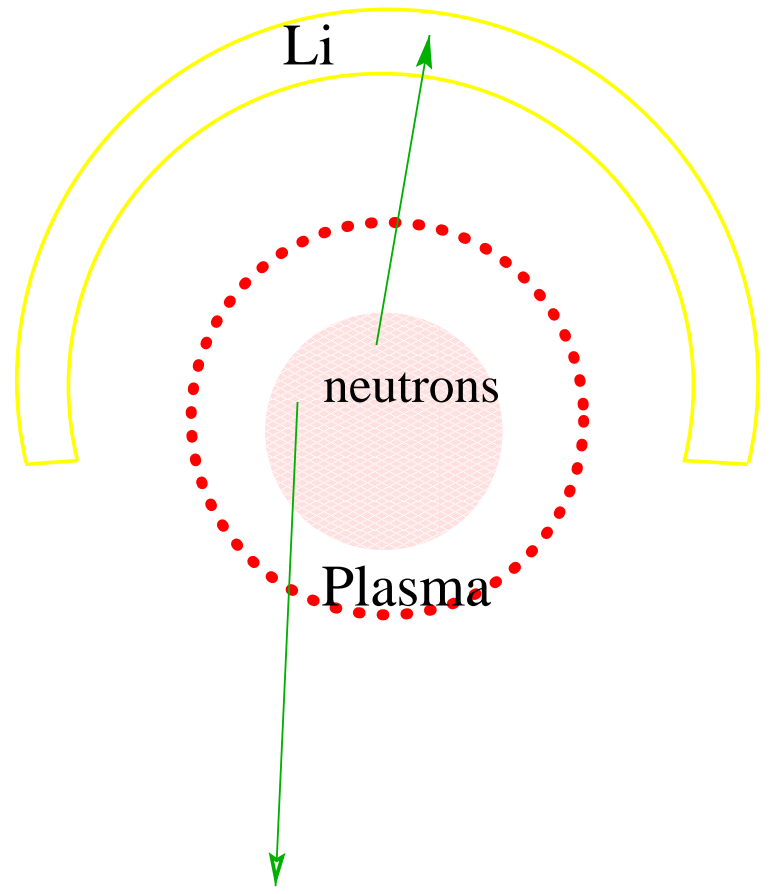

Figure 15: Neutron tokamak based thruster schematic.

estimated as

$$
<v_{\|}>\leq \frac{v_{n 0}}{4}=v_{\alpha 0}=1.3 \times 10^{9} \mathrm{~cm} / \mathrm{sec} .
$$

Here we assumed that only positive momentum is carried by the neutrons. This idea is very simple and does not require any special design of the tokamak. Some loss of the efficiency may be associated with the interaction of the neutrons with the coils.

\section{Summary}

We have proposed a concept of the fusion tokamak powered spaceship. If build it opens new opportunities for the space exploration, such as interplanetary and interstellar rendezvous, implying advanced physical mechanisms not being studied before.

The solution to the problem of deep space propulsion we propose has obvious 
advantages as it relies on the controlled propellant velocity (APV) concept. Expressions for the APV rocket dynamic we obtained can be readily used for the development of the concept once the tokamak reactor design becomes more practical. We can argue that there is a theoretical feasibility of using the fusion tokamak based spaceship.

Our idea of using ripple diffusion for direct particle extraction from the tokamak is new and distinguish our toroidal fusion concept from other tokamak concepts or other fusion devices for the propulsion. We have demonstrated numerically that superthermal particles can be extracted from the tokamak with the statistically dominant direction of their velocity. This is the basis for the developing the fusion propulsion spaceship. Further optimization and more numerical studies are necessary.

New areas of research both experimental and theoretical can be suggested. In the present day experiments the ripple losses are minimized as they deteriorate the plasma confinement and damage the plasma facing components. Thus, use of such mechanism for the thrust creation is the new area of research and needs to be explored in terms of its compatibility with the plasma performance. Another area of research is the mechanisms of controlling the ripple losses, which translates into the control of the thrust. Both approaches, based on designing the magnetic configuration and affecting the kinetics of the particle losses should be investigated.

One of the central problem in any concept based on the thrust by energetic charged particles is associated with compensation of their electric charge when they leave the spacecraft. The advantage of the toroidal device is that this is a minor issue. In the absence of the magnetic field outside the space craft, any electron gun can resolve it. Nevertheless inside the plasma and the fusion reactor, the static charge may produce the well known effect of plasma rotation. It also affects at certain extend the losses of the charged particles and, thus, should be explored in details.

These studies are closely related to the ongoing plasma confinement experimental and theoretical studies, but have a new focus on handling, rather than minimizing, the particle losses for creation the directed thrust. 


\section{Acknowledgments}

We are grateful to S. V. Putvinski, W.J. Emrich, C. H. Williams for useful discussions.

\section{Appendix}

\section{A Rocket and Rocket thrust Equation}

The equation for the evolution of the rocket momentum is determined by the force balance in the laboratory frame:

$$
\frac{d}{d t} V M=\frac{d M}{d t} V+\frac{d V}{d t} M=-M g-\frac{d M}{d t}(v-V),
$$

where $g$ is gravitational (and/or other) acceleration. The thrust is the force exerted by the rocket engine and is given by the rocket thrust equation:

$$
F=-\frac{d M}{d t} v=\frac{d m}{d t} v
$$

To derive rocket equation we rewrite Eq.(21) in the form:

$$
\frac{d \ln M}{d t}=-\frac{g+d V / d t}{v},
$$

which has the following solution, we will call general rocket equation

$$
M(t)=M_{0} \exp \left(-\int_{0}^{t} \frac{g+d V / d t}{v} d t^{\prime}\right) .
$$

\section{A.1 Constant Propellant Velocity (CPV).}

Consider first the case with $v=$ const, when one obtain from Eq.(24) the solution in the form: 


$$
M(t)=M_{0} \exp \left(-\frac{g t+V-V_{0}}{v}\right),
$$

where $V_{0}=V(t=0)$. For our purpose we can further simplify this by assuming that $V \gg V_{0}$. If we estimate required part of flight time in the planet gravitational field, where $g$ is nonzero, as $\Delta t=V_{0} / g$, we also can write this non-equality $g \Delta t / V \ll$ $g \Delta t / V_{0} \ll 1$. Thus we arrive at the conventional rocket equation Eq.(1). This equation can be straightforwardly obtained from Eq.(25) for zero acceleration $g=0$ and initial velocity $V_{0}=0$.

\section{A.2 Adjusted Propellant Velocity (APV).}

For this case generated power can be used most efficiently. For the sake of analytical formulation, we assume $V=\alpha v, \alpha=$ const $<1$. Substituting the propellant velocity in Eq.(24) we obtain the following expression

$$
M(t) \simeq M_{0}\left(\frac{V_{0}}{V}\right)^{\alpha} \exp \left(-\alpha \int_{0}^{t} \frac{g}{V} d t^{\prime}\right)
$$

The space ship can be launched from the earth orbit in which case one should use $g=0$. If the spacecraft is to take off from the earth the integral in Eq.(26) can be estimated as $-\alpha g \Delta t / V_{0}=-\alpha g / \bar{a}$, where $\bar{a}$ is the average acceleration. Again it should be $\bar{a} \gg g$

for the successful launch. This means that the spacecraft spends only a fraction of the flight time in the planet gravitational field. In both cases we result in a modified rocket equation Eq.(2).

\section{References}

[1] Wesson, J., "Tokamaks", Clarendon Press - Oxford, 1997.

[2] Peng, M., "The physics of spherical torus plasmas", Vol. 7, 2000, pp. 1681-1692. 
[3] Williams, C. H., Borowski, S. K., Dudzinski, L. A., Juhasz, A. J., "A Spherical Torus Nuclear Fusion Reactor Space Propulsion Vehicle Concept for Fast Interplanetary Piloted and Robotic Missions", Bull. APS, V.44, N.7, 1999, pp.132-132.

[4] Emrich, W.J., "End Plugging in the Gasdynamic Mirror Using a Field Reversed Configuration", ibid, pp.132-132.

[5] Aymar, R., "ITER-FEAT-the future international burning plasma experimentpresent status", Plasma Phys. Contr. Fusion., Vol.42, Suppl. 12B, 2000, pp.B385B396.

[6] Hawryluk, R. J., et. al., "Results from D-T experiments on TFTR and implications for achieving an ignited plasma", Phi. Trans. R. Soc. Lond. A, Vol. 357, 1999, pp. 443-469.

[7] Kaye, S. M., Bell, M. G., Bell, R. E., et.al. "Initial physics results from the National Spherical Torus Experiment", Phys. Plasmas, Vol. 8, 2001, pp. 1977-1987.

[8] Hosney, A. N., "Propulsion Systems", University of South Carolina Press, Columbia, South Carolina, 1974.

[9] "Propulsion for deep space", Electromagnetic Propulsion Division, Lewis Research Center, NASA, Cleveland, Ohio, 1966.

[10] Zweben, S. J., Darrow, D. S., Herrmann, H. W., et.al., "Measurements of DT alpha particle loss near the outer midplane of TFTR", Nucl. Fusion, V. 35, 1995, pp. 1445-1455.

[11] Goldston, R. J., White, R. B., Boozer, A. H., "Confinement of high-energy trapped particles in tokamaks", Phys. Rev. Lett., V. 47, 1981, pp. 647-649.

[12] Morozov, A. I., Solov'ev, L. S., "Motion of charged particles in electromagnetic fields", in Reviews of Plasma Physics, Vol.2 (Acad. Leontovich, M. A., Ed.), Consultants Bureau, New York, 1966, pp. 201-297. 
[13] Zakharov, L. E., Shafranov, V. D., "Equilibrium of current-carrying plasmas in toroidal configurations", ibid., V. 11, 1981, pp. 153-302.

[14] Zakharov, L. E., "Tokamak with lithium covered walls", Bull. APS, V.45, N.7, 2000, pp.225-225. 


\section{External Distribution}

Plasma Research Laboratory, Australian National University, Australia

Professor I.R. J ones, Flinders University, Australia

Professor J oão Canalle, Instituto de Fisica DEQ/IF - UERJ , Brazil

Mr. Gerson O. Ludwig, Instituto Nacional de Pesquisas, Brazil

Dr. P.H. Sakanaka, Instituto Fisica, Brazil

The Librarian, Culham Laboratory, England

Library, R61, Rutherford Appleton Laboratory, England

Mrs. S.A. Hutchinson, JET Library, England

Professor M.N. Bussac, Ecole Polytechnique, France

Librarian, Max-Planck-Institut für Plasmaphysik, Germany

J olan Moldvai, Reports Library, MTA KFKI-ATKI, Hungary

Dr. P. Kaw, Institute for Plasma Research, India

Ms. P.J . Pathak, Librarian, Insitute for Plasma Research, India

Ms. Clelia De Palo, Associazione EURATOM-ENEA, I taly

Dr. G. Grosso, Instituto di Fisica del Plasma, Italy

Librarian, Naka Fusion Research Establishment, J AERI, J apan

Library, Plasma Physics Laboratory, Kyoto University, J apan

Research Information Center, National Institute for Fusion Science, J apan

Dr. O. Mitarai, Kyushu Tokai University, J apan

Library, Academia Sinica, Institute of Plasma Physics, People's Republic of China

Shih-Tung Tsai, Institute of Physics, Chinese Academy of Sciences, People's Republic of China

Dr. S. Mirnov, TRINITI, Troitsk, Russian Federation, Russia

Dr. V.S. Strelkov, Kurchatov Institute, Russian Federation, Russia

Professor Peter Lukac, Katedra Fyziky Plazmy MFF UK, Mlynska dolina F-2, Komenskeho Univerzita, SK-842 15 Bratislava, Slovakia

Dr. G.S. Lee, Korea Basic Science Institute, South Korea

Mr. Dennis Bruggink, Fusion Library, University of Wisconsin, USA

Institute for Plasma Research, University of Maryland, USA

Librarian, Fusion Energy Division, Oak Ridge National Laboratory, USA

Librarian, Institute of Fusion Studies, University of Texas, USA

Librarian, Magnetic Fusion Program, Lawrence Livermore National Laboratory, USA

Library, General Atomics, USA

Plasma Physics Group, Fusion Energy Research Program, University of California at San Diego, USA

Plasma Physics Library, Columbia University, USA

Alkesh Punjabi, Center for Fusion Research and Training, Hampton University, USA

Dr. W.M. Stacey, Fusion Research Center, Georgia Institute of Technology, USA

Dr. J ohn Willis, U.S. Department of Energy, Office of Fusion Energy Sciences, USA

Mr. Paul H. Wright, Indianapolis, Indiana, USA 
The Princeton Plasma Physics Laboratory is operated by Princeton University under contract with the U.S. Department of Energy.

\author{
Information Services \\ Princeton Plasma Physics Laboratory \\ P.O. Box 451 \\ Princeton, NJ 08543
}

Phone: 609-243-2750

Fax: 609-243-2751

e-mail: pppl_info@pppl.gov

Internet Address: http://www.pppl.gov 